\title{
Modelling Artificial Immune - Tumor Ecosystem Interaction During Radiation Therapy Using a Perceptron - Based Antigen Pattern Recognition
}

\author{
Stephan Scheidegger ${ }^{1}$, Alexander Mikos ${ }^{1}$ and Harold Fellermann ${ }^{2}$ \\ ${ }^{1}$ ZHAW School of Engineering, Zurich University of Applied Sciences, Winterthur, Switzerland \\ ${ }^{2}$ Interdisciplinary Computing and Complex Biosystems Research Group, School of Computing, Newcastle University, Newcastle \\ upon Tyne, UK \\ scst@zhaw.ch
}

\begin{abstract}
The dynamics of an artificial tumor-immune - ecosystem after simulated radiation therapy (RT) was investigated. The system is represented by a model for a tumor - host-tissue system including repopulation, mutation, competition and interaction with antibodies and a perceptron used for antigen pattern recognition. The perceptron response governs the generation of antibodies. The system exhibit interesting dynamic aspects: A special focus of the presented work lies on the observed separation of the perceptron weights for tumor - and host tissue, After RT application, the weights for host tissue can evolve into negative values whereas tumor-related perceptron weights remain positive. The negative perceptron weights indicate an immune-suppressive effect after RT which is related to the host tissue.

The applicability of the presented system to clinical treatment optimization is not possible and may remain strongly limited when refined. The matching with a real-world tumorimmune-ecosystem (in patient) is questionable and the chosen approach may be too simplistic. However, the idea of an immune system considered as a trainable perceptron offers new hypothesis for novel approaches to anti-cancer treatments, treatments of infectious diseases or even vaccination.
\end{abstract}

\section{Introduction}

Radiation Therapy (RT) remains one of the central pillars of anti-cancer treatment. However, there are several challenges in understanding the biological processes behind the different effects of RT: First, the differences between the behavior of tumor cells in vitro, in vivo and in patient (clinical situation) prevent a direct transfer of knowledge elaborated by experiments in vitro or in vivo (mouse model) to clinical treatment. Several studies hypothesize that the major cause of radio-resistance observed during $\mathrm{RT}$ treatments may be related to the heterogeneity of tumor tissues (Horsman MR et al., 2012; Baumann et al. 2016). In addition, the role of patientspecific reaction of the immune system during or after anticancer treatment often remains unclear and is recently investigated (Fridman et al. 2017).

Second, monitoring relevant aspects of the dynamic tumor response in patient would require time resolved observations including advanced imaging techniques and tracking of immune status by analyzing blood - and tissue samples (biopsy material). Radiation-triggered processes take place on different scale levels of the biological system "patient" (Fig.1): On every scale, typical biological observations may serve as "data sources" allowing the exploration of specific aspects of the system, ranging from molecular level (signaling pathways) up to the patient as a very complex system. In principle, these biological observations may be compared to the output of models in silico. Models on the molecular scale can be formulated as chemical reaction-diffusion system. This becomes difficult for high level-models and therefore, alternate approaches are required. An interesting possibility to deal with the enormous complexity of biological systems could be based on the idea of mimicking the "biological semantics" by a mathematical description instead of describing the molecular (chemical) or physical processes directly (Scheidegger et al., 2014). Such an approach is more referring to the system behavior regarding its meaning than the way how exactly information is processed or stored by molecules (syntax).

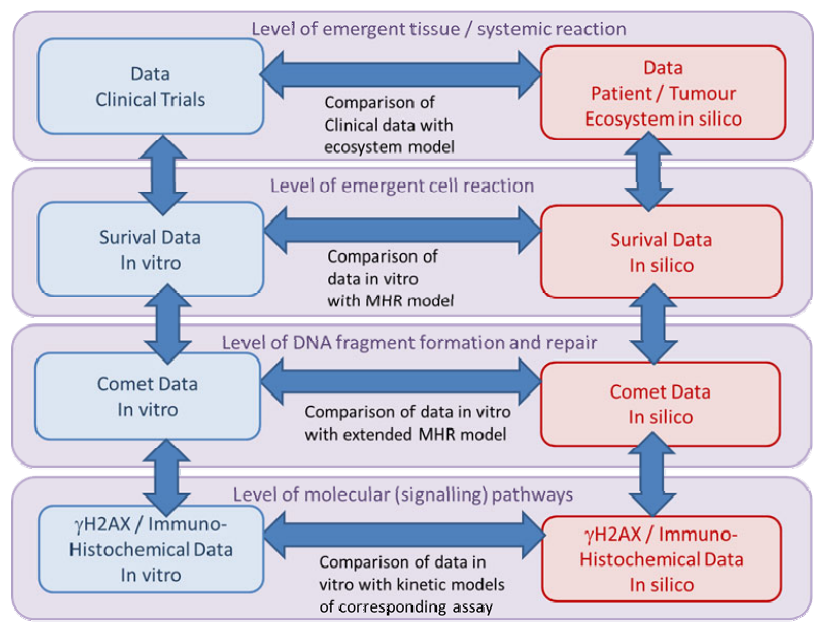

Figure1. Different levels (scales) of the patient as a system: On molecular and cellular scale, $\gamma \mathrm{H} 2 \mathrm{AX}$ - and Comet assays serve as examples. 
The big challenge to catch the semantics with an appropriate model is related to the plasticity of biological systems which leads to a broad spectrum of different adapted responses. Sematic models may be restricted to a narrow range of dynamic conditions and therefore may have limited predictive power. However, artificial systems inspired by biological semantics may help to discover characteristic dynamic patterns which can be compared to the observations derived by experiments in vitro, in vivo or in patient.

In principle, a tumor can be considered as an ecosystem with different interacting cell populations (different tumor sub-clones, host tissue including tumor-associated fibroblasts, endothelial cells / vascular system, immune cells etc.). Radiation-induced cell killing may influence competition, commensalism, repopulation and immune cell interaction ("special form of predation") and can be regarded as a selective process that leads to survival of radio-resistant subpopulations. Radiation-induced loss of competition between the different cancer sub-populations may lead to an accelerated progress of disease, which would be a very unwanted effect of RT. However, investigating tumor ecosystem dynamics including immune system interaction seems worth to be explored by artificial systems in silico since the access to time-resolved information about the "real-world" biological system is very difficult on the scale of patients and clinical trials.

Evolutionary dynamics of cancer (Crespi \& Summers, 2005) could be responsible for an adapted response of the tumor to anti-cancer therapy. The discussion of ecological and evolutionary aspects of anti-cancer therapy is not new and has been discussed by different authors (Pienta et al., 2008; Basanta et al. 2015; Basanta et al., 2012; Merlo et al., 2006). Gatenby et al. (2010) discussed the role of evolutionary dynamics for cancer prevention. Ecological principles have also been considered for investigating metastasizing cancer cells (Chen \& Pienta, 2011). Meta-population dynamics regarding spatial heterogeneity have been investigated by Gonzáles-Garcia et al. 2002. There seems to be certain evidence that tissue interactions might play a pivotal role in development and therapy response of tumors. The ecosystem perspective to a tumor may elucidate cancer dynamics in patient and therefore could help to improve or optimize anticancer treatment.

A system-level model for the tumor - immune system response onto RT has been presented by Scheidegger and Fellermann (2019). In this study, only an innate-type response of the immune system was considered. The inclusion or extension to an adaptive immune response would require an antigen pattern recognition process. Due to the shear complexity of the immune system, we regard it as unpromising to bring details of the molecular interactions into a computer model: Many needed parameter values are unknown and may be patient specific. Quantifiable biological experiments in vitro or in vivo (mouse model) may not represent the real dynamics in a specific patient. Accessing time-resolved data during clinical treatment or trials is very limited. Therefore, we propose a system dynamics - based semantic model of an artificial adaptive immune system in order to support hypothesis generation and design of experiments in vitro or clinical trials. Banda et al. (2013) proposed an implementation of a perceptron in a chemical system. The feasibility of a realization of a perceptron in a chemical (and based on this in a biological) system and the way how the immune system processes potential antigens by Antigen Presenting Cells (APC) led to the hypothesis that the semantics of the adaptive immune system may be described by a perceptron. A special emphasis in this work was set to the influence of the ecosystem dynamics onto the evolution of the perceptron weights as a characteristic feature of the combined antigen - danger signal recognition.

\section{Materials \& Methods}

The model used for investigating the dynamic response of a combined tumor - immune ecosystem onto radiation therapy (RT) consists of two sub-models: The ecosystem sub-model captures processes such as repopulation, mutation, competition, cell death (radiation and immune system induced), antigen-antibody interaction and danger signal generation. The immune system sub-model captures the plasticity of the immune system, which enables its adaptability and ability to learn, along with molecular danger signals and antigen-antibody interactions. In the absence of detailed molecular knowledge and in order to focus on its "biological semantics", the immune response is here represented by a simple perceptron-based pattern-recognition system.

The coupling between the two sub-models is mediated by an antigen pattern vector. In this artificial system, nine vector components ( $\vec{X}=X_{i}$ with $i=1 \ldots 9$ ) are selected, regardless of their specific meaning or correspondence to the real biological system. It is assumed that every tumor - cell population (sub-population or sub-clone) and the host tissue population has its own characteristic pattern (pattern vector $\vec{P}_{k l}$ with components $=1$ for bearing a specific antigen corresponding to the antigen pattern vector component $X_{i}$ and 0 otherwise). The assignment to the nine tumor-subpopulations and the host tissue is illustrated in Fig.2.

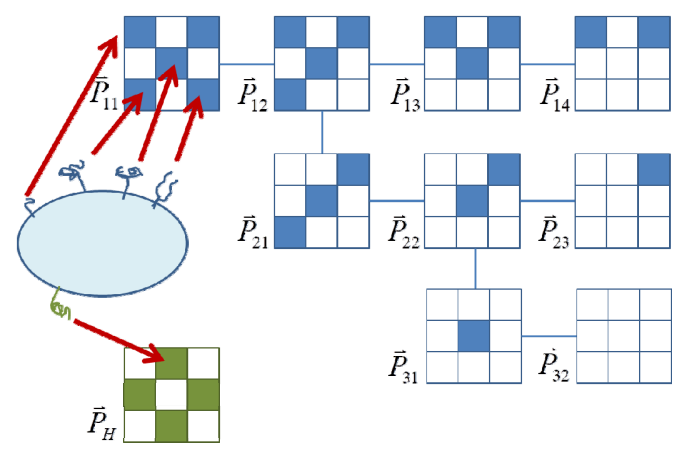

Figure 2. Antigen pattern representation: The antigen pattern vector consists of 9 elements (displayed as a 3 × 3 matrix). Every element is intended to correspond to a specific antigenic structure. It is important to note that an argument for the orthogonal choice for the host tissue could be the fact that tumorspecific molecules may not be considered as self, but following the danger model from Matzinger (2002), more important for immune-system-mediated response may be association of these molecules with a danger signal. 
With every mutation, it is assumed that the tumor cells are removing components of the antigen pattern vector (eliminating the expression of the corresponding molecules). This scenario mimics an immunological escape (Vesely et al. 2011) which may be based on reduction of self-antigen presenting Class I Major Histocompability Complex MHC (Dunn et al. 2002). To limit the complexity of the model, we selected three branches (mutation lines, $k=1 \ldots 3$ ) of mutation-driven antigen elimination, resulting in nine tumor sub-clones and a total of 10 populations (figure $2 \& 3$ ). The value of the antigen pattern vector components $X_{i}$ is dependent on the presence of corresponding cells and is assumed to follow a sigmoid relationship, e.g. the $X_{1}$ is (according to figure 2) given by:

$$
X_{1}=\frac{\left(T_{11}+T_{12}+T_{13}+T_{14}\right)^{2}}{\left(X_{a c t}\right)^{2}+\left(T_{11}+T_{12}+T_{13}+T_{14}\right)^{2}}
$$

Following the danger model from Matzinger (2002), the immune response is only activated when a danger signal is present. The detection of simultaneous danger signal - and antigen pattern presence is modelled by a perceptron, which sums the weighted antigen signal:

$$
\Sigma=\sum_{i=1}^{9} w_{i} X_{i}
$$

The weights evolve by reinforcement learning. For every perceptron weight $w_{i}$, the calculation of the weight is given by the following differential equation:

$$
\frac{d w_{i}}{d t}=a \cdot(D-Y) \cdot X_{i}
$$

$D$ is denoting the danger signal and $Y$ represents the following, sigmoidal response to the sum of weighted antigen signals $\Sigma$ :

$$
Y=\frac{\Sigma^{\xi}}{Y_{a c t}^{\xi}+\Sigma^{\xi}}
$$

The danger signal is assumed to be generated by cell death. Appearance of these danger signals and corresponding antigen patterns is based on the ecosystem part of the model (Fig.3, blue box). In the model, the different populations can grow (growth rate constant $k_{T k l}$ for tumor and $k_{a H}$ for host tissue), interact (competition, interaction constants $k_{T T}$ for tumortumor competition, $k_{T H}$ and $k_{H T}$ for tumor-host tissue interaction), mutate (only tumor sub-clones, mutation rate constant $k_{m u t}$ ) or can be eliminated. Beside a spontaneous elimination process (elimination rate constant $k_{e T}$ ) radiationinduced cell killing is implemented by a dynamic linearquadratic model ( $\Gamma$-LQ-model; Scheidegger et al., 2011a,b) using the radiation sensitivity coefficients $\alpha_{T}$ and $\beta_{T}$ for tumor cells respectively $\alpha_{H}$ and $\beta_{H}$ for host tissue. This model uses a Transient Biological Dose Equivalent (TBDE) $\Gamma$, which includes cellular repair by a kinetic model:

$$
\frac{d \Gamma}{d t}=R-\gamma \Gamma
$$

For a fixed radiation dose rate $R$, the repair parameter $\gamma$ can be assumed to be constant. Summing up all these processes, a system of ordinary differential equations describing the temporal changes of the population sizes for tumor cells $\left(T_{i k}\right)$ and host tissue $(H)$ can be formulated:

$$
\begin{aligned}
& \frac{d T_{11}}{d t}=\left(k_{T 11}-k_{m u t}-k_{e T}-r_{11} k_{I T}-k_{H T} H-k_{T T} T-\left(\alpha_{T}+2 \beta_{T} \Gamma\right) \cdot R\right) \cdot T_{11} \\
& \frac{d T_{i k}}{d t}=\left(k_{T i k}-k_{e T}-r_{i k} k_{I T}-k_{H T} H-k_{T T} T-\left(\alpha_{T}+2 \beta_{T} \Gamma\right) \cdot R\right) \cdot T_{i k}+k_{m u t} \cdot q_{i l} T_{l k} \\
& \frac{d H}{d t}=\left(k_{a H}-k_{e H}-r_{H} k_{I H}-k_{b H} H-k_{T H} T-\left(\alpha_{H}+2 \beta_{H} \Gamma\right) \cdot R\right) \cdot H
\end{aligned}
$$

$T$ is the total amount of tumor cells overall sub-clones ( $T=\sum_{i, k} T_{i k}$ ) and $q_{i l}$ is a matrix representing the topology of the population network (with branches or chains $i$ and elements $k$ ) describing the mutations and sub-clone generation (by the rate constant $k_{m u t}$ ): For a population in a chain (e.g. $T_{13}$, see figure 3), $q_{i l}=-1, q_{i, l-1}=1$, for a "node" (e.g. $\left.T_{12}\right), q_{i+1, l}=-1$. The factor $r_{i k}$ includes the interaction with the adaptive immune system which will be explained later. To get a danger signal $D$, a corresponding process (inflammation) must be triggered. It is assumed that this will be mediated by cell death (especially necrosis). Therefore, the amount of necrotic cells $N$ is calculated in the model as follows:

$$
\begin{aligned}
& \frac{d N_{11}}{d t}=\left(k_{e T}+r_{11} k_{I T}+\left(\alpha_{T}+2 \beta_{T} \Gamma\right) \cdot R\right) \cdot T_{11}-k_{n} N_{11} \\
& \frac{d N_{i k}}{d t}=\left(k_{e T}+r_{i k} k_{I T}+\left(\alpha_{T}+2 \beta_{T} \Gamma\right) \cdot R\right) \cdot T_{i k}-k_{n} N_{i k} \\
& \frac{d N_{H}}{d t}=\left(k_{e H}+r_{H} k_{I H}+\left(\alpha_{H}+2 \beta_{H} \Gamma\right) \cdot R\right) \cdot H-k_{n} N_{H}
\end{aligned}
$$

The danger signal is calculated according by a sigmoidal relation:

$$
D=\frac{\left[\sum_{i, k} N_{i k}+h \cdot N_{H}\right]^{2}}{L_{a c t}^{2}+\left[\sum_{i, k} N_{i k}+h \cdot N_{H}\right]^{2}}
$$


The factor $h$ is introduced to govern the ratio of necrotic and apoptotic cell death. Some tumors lose the ability to undergo apoptosis and the necrotic pathway will be the main elimination process. In contrast, host tissue may be eliminated by necrotic or apoptotic cell death. Apoptotic cell death may not be equally considered as a danger signal compared to necrotic cell death (Grimsley and Ravichandran, 2003), where for example a release of intracellular Heat Shock Proteins (HSP, Daugaard et al., 2007) may be involved (Srivastava, 2002). Therefore, the contribution of host tissue cell killing to the danger signal should be weighted according the ratio of apoptotic / necrotic cells. This is a simplistic approach of a complex system involving processing danger signals and Damage - Associated Molecular Patterns (DAMP) by Antigen-Presenting Cells (APC).

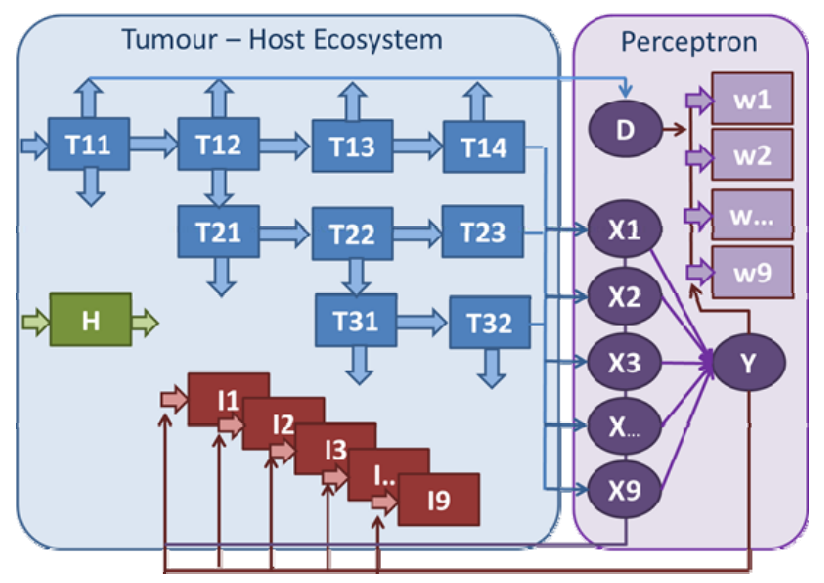

Figure 3. Schematic illustration of the model structure: For clarity, not all connections and flows are shown. Integrators are depicted by rectangles with the corresponding flows as thick arrows; thin arrows and lines are indicating the flow of information. The ecosystem part can be considered as compartment containing the tumor cell populations $T_{i k}$, the host cell population $H$ and an antibody population $I_{n}$ (or receptor population located at the surfaces of immune cells). Interactions (competition, predation) between the cell populations are not depicted. The perceptron is a more a functional unit than a compartment, $D$ is the "desired" or "danger" signal, the vector $X_{n}$ represents the antigen pattern and $Y$ is the perceptron response. The weights $w_{n}$ are used for summing up the antigen pattern signal.

The generation of the number of antibodies $I_{n}$ is governed by the perceptron response $Y$ and the presence of corresponding antigen pattern vector components $X_{n}$. In this model, antibodies can be eliminated spontaneously (by the elimination rate $k_{e I} \cdot I_{n}$ ) or by an antigen-antibody-interaction. It is important to note that in this model, the probability of an antigenantibody interaction is dependent on the fit of the antigen pattern to the antibody pattern. The assumption behind this is that the immune cells are evaluating the antigen-pattern as a whole and interact stronger, when a good fit is reached. Following this idea, the immune response is triggered dependent on the dot product between the antigen-pattern vector $\vec{P}_{i k}$ with the antibody pattern vector $I_{n}=\vec{I}$ expressed by the perceptron-mediated response (system of APC, Thelper cells, B-cells etc.):

$$
r_{i k}=\vec{I} \bullet \vec{P}_{i k}
$$

According to this, the production and elimination of the antibody population can be described by:

$$
\frac{d I_{n}}{d t}=k_{I} Y X_{n}-\left(k_{e I}+\left(\alpha_{I}+2 \beta_{I} \Gamma\right) \cdot R\right) \cdot I_{n}-k_{I T} \cdot\left(\sum_{i, k} r_{i k} T_{i k}\right)_{n}
$$

The radiation sensitivity coefficients $\alpha_{I}$ and $\beta_{I}$ reflect the fact that the immune response is mediated by more or less radiation sensitive immune cells, which are irradiated in the tumor compartment. For simulation, a duration of 1200-1500 $\mathrm{d}$ (with numerical time increments of $\Delta t=10^{-3} \mathrm{~d}$ and a 4th order Runge-Kutta integration) was chosen. The results depend on the initial values and the "pre-evolution" of the system (evolution before application of RT). All calculations are performed with the initial conditions $T_{i k}=0$ except $T_{11}$ $=10^{-3}\left(=10^{6}\right.$ cells $)$ and a start of RT treatment at day 570 . The model parameters are summarized in table 1 .

It is worth emphasizing that in this work, the dynamics of an artificial system is investigated and that the used parameter values are describing an artificial model and may not correspond to a value assessed form a real biological system. Exceptions are the radiation sensitivity coefficients which are known for a large number of tissues and tumors (e.g. van Leeuwen et al. 2018) or tumor doubling times (Steel, 1977; Mehrara et al., 2007).

The radiation therapy scheme in principle consists of 3 fractions with a radiation dose of 2 Gy per fraction and a time interval of 2 days between the fractions, followed by daily fractions. This scheme has turned out to be more effective than daily RT applications when considering innate immune response in silico (Scheidegger and Fellermann, 2019). In this work, we modified this fractionation (different dose values per fractions and varying number of fractions) to investigate the effect of RT onto the dynamics of the system.

\section{Results}

The development of the tumor populations and perceptron weights after application of 3 fractions with 2 Gy is shown in figure 4: Before the irradiation, the tumor cell populations are growing and mutating. After 400 days, the host tissue population decreases rapidly due to competition with the growing tumor. After application of RT, the host tissue repopulates. The host tissue is considered to be less radiosensitive and therefore is less affected compared to tumor cells. The subsequent aggressive regrowth of the tumor and competition suppresses repopulation of host tissue after $660 \mathrm{~d}$. 


\begin{tabular}{|c|c|c|}
\hline Parameter / unit & \multicolumn{2}{|l|}{ Description and default value } \\
\hline$k_{T 11}=k_{T i k} / \mathrm{d}^{-1}$ & Tumor growth rate constant & $3.46 \cdot 10^{-2}$ \\
\hline$k_{m u t} / \mathrm{d}^{-1}$ & mutation rate constant & $10^{-3}$ \\
\hline$k_{e T} / \mathrm{d}^{-1}$ & $\begin{array}{l}\text { tumor cell elimination rate } \\
\text { constant }\end{array}$ & $10^{-2}$ \\
\hline$k_{T T} / \mathrm{d}^{-1}$ & tumor cell growth inhibition & $10^{-4}$ \\
\hline$k_{I T} / \mathrm{d}^{-1}$ & immunogenic elimination & $10(1)$ \\
\hline$k_{H T} / \mathrm{d}^{-1}$ & $\begin{array}{lll}\begin{array}{l}\text { Host } \\
\text { interaction }\end{array} & \text { tumor } & \text { cells } \\
\end{array}$ & $10^{-3}$ \\
\hline$k_{T H} / \mathrm{d}^{-1}$ & $\begin{array}{llll}\begin{array}{l}\text { Tumor } \\
\text { interaction }\end{array} & \text { host } & \text { cells } \\
\end{array}$ & $3 \cdot 10^{-2}$ \\
\hline$k_{a H} / \mathrm{d}^{-1}$ & host cell growth & $10^{-1}$ \\
\hline$k_{b H} / \mathrm{d}^{-1}$ & host cell growth inhibition & $9.7 \cdot 10^{-3}$ \\
\hline$k_{e H} / \mathrm{d}^{-1}$ & host cell elimination & $10^{-5}$ \\
\hline$k_{n} / \mathrm{d}^{-1}$ & Necrotic cell elimination & 10 \\
\hline$k_{I H} / \mathrm{d}^{-1}$ & immunogenic elimination & $10^{-3}(1)$ \\
\hline$k_{I} / \mathrm{d}^{-1}$ & immune cell production & 10 \\
\hline$k_{e I} / \mathrm{d}^{-1}$ & immune cell elimination & 1 \\
\hline$k_{M} / \mathrm{d}^{-1}$ & memory cell production & $10^{-2}$ \\
\hline$Y_{a c t}$ & $\begin{array}{l}\text { danger signal activation } \\
\text { level }\end{array}$ & 3 \\
\hline$\xi$ & & 9 \\
\hline$X_{a c t}$ & Pattern recognition level & 2 \\
\hline$h$ & $\begin{array}{l}\text { weight for danger signal } \\
\text { contribution }\end{array}$ & variable \\
\hline$L_{a c t}$ & Danger signal param. (eq.7) & 1 \\
\hline$a / 1 \mathrm{~d}^{-1}$ & perceptron weight & 1 \\
\hline$\alpha_{T} / G y^{-1}$ & $\begin{array}{l}\text { Radiation sensitivity } \\
\text { coefficient tumor cells }\end{array}$ & 0.3 \\
\hline$\beta_{T} / G y^{-2}$ & $\begin{array}{l}\text { Radiation sensitivity } \\
\text { coefficient tumor cells }\end{array}$ & 0.06 \\
\hline$\alpha_{H} / G y^{-1}$ & $\begin{array}{l}\text { Radiation sensitivity } \\
\text { coefficient host tissue }\end{array}$ & 0.1 \\
\hline$\beta_{H} / G y^{-2}$ & $\begin{array}{l}\text { Radiation sensitivity } \\
\text { coefficient host tissue }\end{array}$ & 0.05 \\
\hline$\gamma / \mathrm{d}^{-1}$ & Radiobiol. repair constant & 4 \\
\hline
\end{tabular}

Table 1: Model parameter values used for simulations: The parameter values $\left(k_{T T}, k_{I T}, k_{H T}, k_{T H}, k_{b H}, k_{I H}, L_{a c t}\right)$ are normalized to $10^{9}$ cells $(=1)$. Values in brackets are selected for alternatives scenarios, some of the parameters values have been varied (s. figures 5-9).

The population $T_{32}$ does not bear any antigen pattern and grows to the equilibrium level $T_{32, e q}$ defined by the ratio of the net growth rate constant and the competition / interaction strength (described by $k_{\mathrm{TT}}$ ):

$$
T_{32, e q}=\frac{k_{T 32}-k_{e T}}{k_{T T}}
$$

For the parameter values given in table 1, the equilibrium level is $246\left(2.4610^{11}\right.$ cells $)$. This is in full agreement with the observed equilibrium level in the simulation (approx. after 1500 days, not shown in the diagram figure 4a). After day 848 , a strong and fast decrease of the tumor populations can be observed without any irradiation. This event coincides with rapidly increasing perceptron weights for the odd (tumorassociated) antigen vector components (figure $4 b$ ). At this time, the process is triggered by increasing cell death in the tumor populations and the subsequent generation of the danger signal.
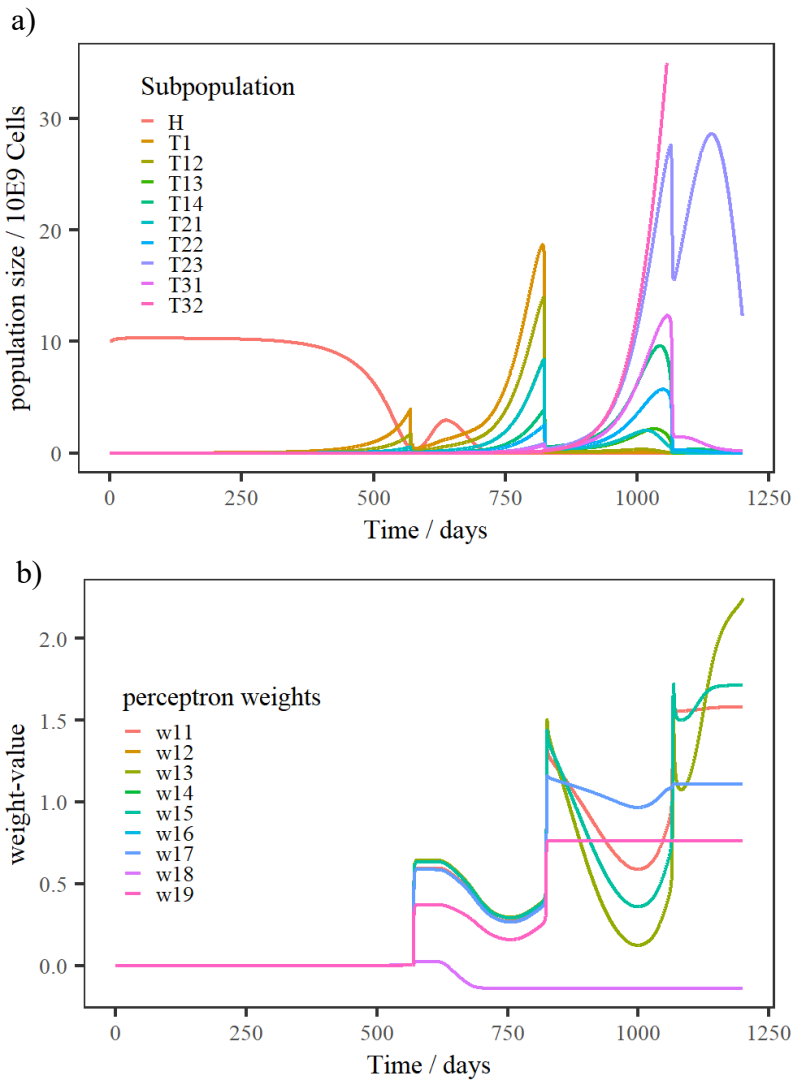

Figure 4. Development of the cancer cell and host tissue populations (a) and the perceptron weights (b) before and after RT ( $3 \times 2$ Gy, application of fractions on day 570, 572, 574): Time axis is given in days. The curves for the even weights are identical with $w_{14}$.

A remarkable phenomenon can be observed for the even components of the antigen pattern vector: Approximately 54 days after application of the last RT fraction, the even weights start to evolve to negative values. According equation (1), these weights are negative contributions to the weighted sum of the antigen pattern and reduce the perceptron response $Y$ (equation 3). The evolution of the weights is a result of the ecosystem dynamics combined with reinforced learning of the perceptron. The even weight values remain negative since the host tissue (and the corresponding even antigen pattern vector 
components) disappear after day $800\left(H<10^{-9}\right.$ or less than 1 cell). The odd weights (tumor weights) decrease parallel to the even weights (host weights) but as a result of the competition between host tissue and tumor, more cells remain due to repopulation long after RT application. The appearance of negative values can only be observed for a radiation dose of more than 1 Gy per fraction (figure 5).
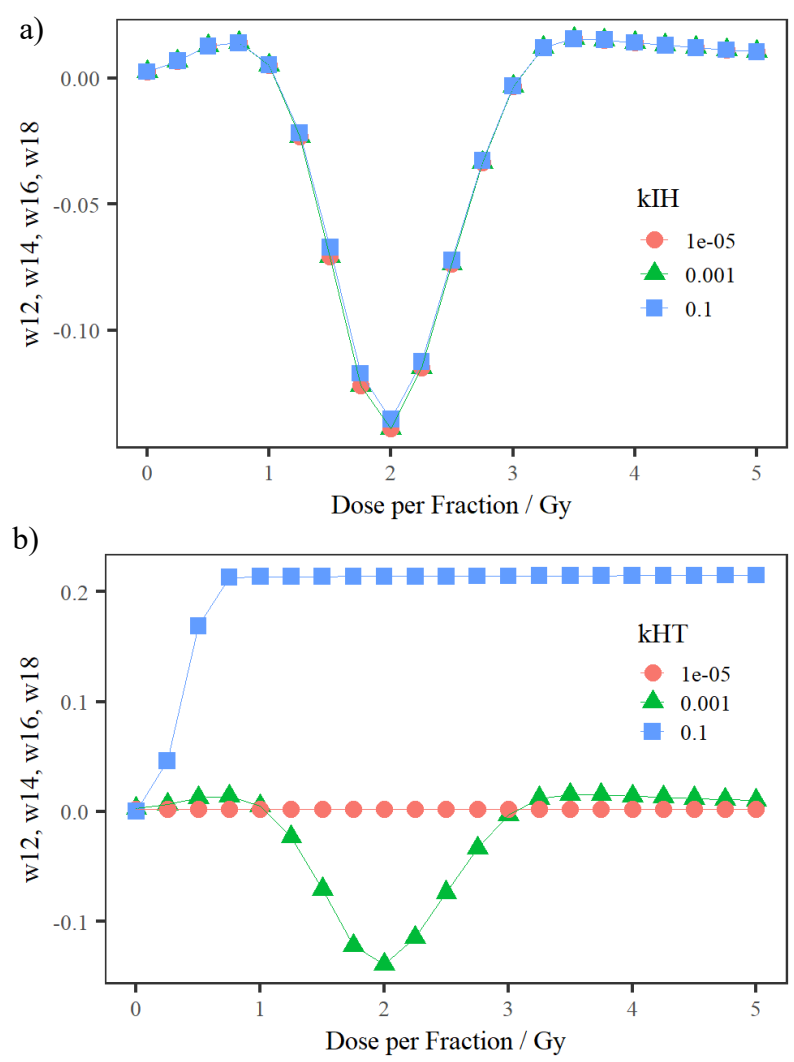

Figure 5. Perceptron weights of the even components (host weights) of the pattern vector $(i=\{2,4,6,8\})$ as function of the radiation dose per fraction for (a) different values of $k_{I H}$ and (b) different values for $k_{H T}$ : Values in equilibrium (after host tissue population and corresponding antigen pattern have vanished and according equation 3 , no further modifications of weights occur), calculated for $3 \times 2 \mathrm{~Gy}$.

In figure 6 , the host perceptron weights are displayed as a function of $h$ : The factor $h$ was introduced to weight the host tissue contribution to the danger signal. A large value of $h$ is associated with more immune system activation. Biologically, this may be interpreted as increased necrotic cell death whereas a small value of $h$ may represent apoptotic tissue. Dependent on the radiation dose per fraction, even for $h=10$, the perceptron weights can evolve into negative values.

After reaching the equilibrium (due to a lack host tissue cells and therefore the absence of the even components of the antigen pattern vector), the final host weights are dependent on the host-tumor interaction. For $k_{H T}=k_{T T}=10^{-4}$, the weights do not evolve into negative values, whereas for larger values of $k_{I H}$, the weights become negative but below $k_{H T}=9.8 \cdot 10^{-4}$ independent of $h$. Above this limit, the host weights exhibit a $h$-dependence. Interestingly, this behavior is similar to a nonorthogonal pattern, where the host tissue cells bear the tumorrelated antigen pattern vector component $X_{5}$. In addition, the evolution into negative weights can be observed over a large range of varying parameters: In the standard settings in table 1 , the interaction of host tissue with the immune system is weak compared to the tumor cells. The rational for this is that similar host tissue may be found outside of the tumor compartment, resulting in an additional suppression of an immune response against host tissue cells. Interestingly, negative host tissue perceptron weights can be found even for similar interaction strength with the immune system as for tumor cells (blue stars in figure 6).

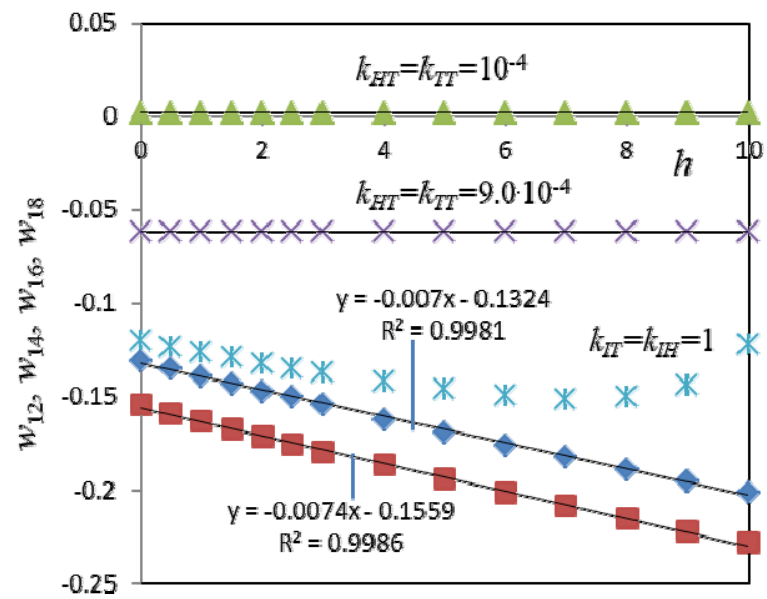

Figure 6. Perceptron weights of the even components of the pattern vector $(i=\{2,4,6,8\})$ as function of $h$ at the equilibrium: Blue diamonds are calculated for $k_{H T}=10^{-4} \mathrm{~d}^{-1}$ according table 1; red squares represent the non-orthogonal case, where host tissue cells bear the antigen vector component $X_{5}$. The even weights are strongly dependent on the host-tumor competition parameter $k_{H T}$, the weights are $h$ - independent for values below $9.8 \cdot 10^{-4} \mathrm{~d}^{-1}$ whereas above, the linear relationship exhibit a reduction of the even weights for increasing $h$. Blue stars indicate a case, where host tissue cells and tumor cells have the same interaction strength with the immune system; calculated for $3 \times 2$ Gy.

In figure 7, the effect of subsequent RT fractions is shown: In agreement with figure 5 , the host weights evolve to negative values above $3 \mathrm{~Gy}(3 \times 1 \mathrm{~Gy}$ in figure corresponds to 1.5 fractions with $2 \mathrm{~Gy}$ in figure 7). The weights remain negative for higher dose values. With $3 \times 2$ Gy, the tumor cells repopulate and due to the competition between tumor cells and host tissue, the number of host tissue cells drop below $10^{-9}$ ( 1 cell) on day 772, and therefore, no further modification of the even weights takes place. Higher radiation dose in combination with the higher radiation sensitivity of tumor cells leads to a modification of the competition between host tissue and tumor 
cells, resulting in a slight increase of the even weights with rising dose.

Varying values of the repair constant (figure 8) are dependent on the host - tumor competition, influencing the evolution of the weights below $\gamma=3 \mathrm{~d}^{-1}$ : Such values correspond to incomplete repair. Above this threshold, complete repair occurs (an irrelevant small amount of $\Gamma$ remains for the subsequent fraction) and therefore repair speed does not influence the outcome.

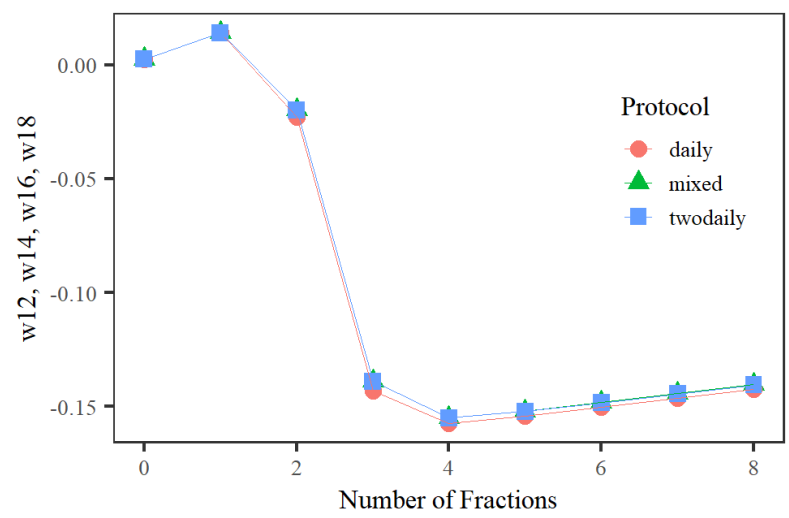

Figure 7. Even perceptron weights as a function of the number of 2 Gy - RT fractions with daily application of 2 Gy, first 3 fractions with a time gap of 2 days, subsequent fractions daily (mixed) or two days interval between all fractions (of $2 \mathrm{~Gy}$ ).

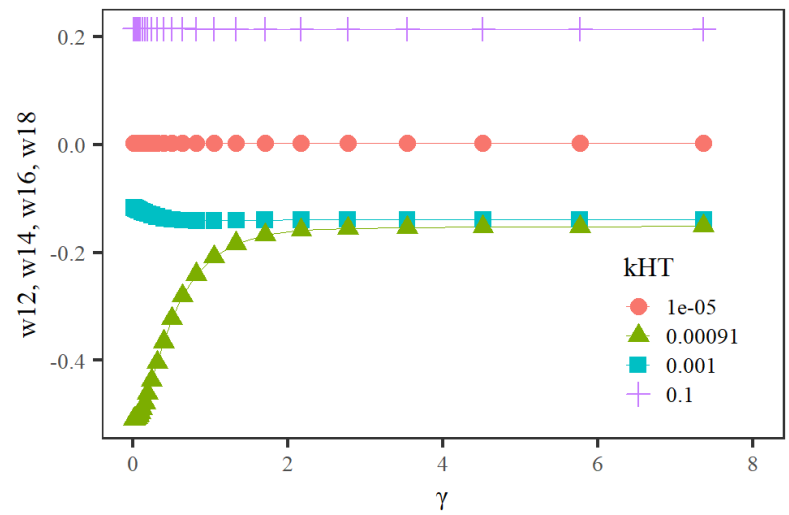

Figure 8. Even perceptron weights as a function of the radiobiological repair constant $\gamma$ and different host-tumor competition strength.

Further investigations of the model's dependency from the competition parameters between host-tissue and tumor cells $\left(k_{H T}, k_{T H}\right)$ and tumor-tumor competition $\left(k_{T T}\right)$ show a highly sensitive behavior in a certain range of the parameter values, which seems to be individual for the three different parameters (Figure 9). Especially for $k_{H T}$, the even weights jump over very small parameter range around $10^{-3}$, but remain stable below $2 \cdot 10^{-4}$ and above $10^{-2}$.

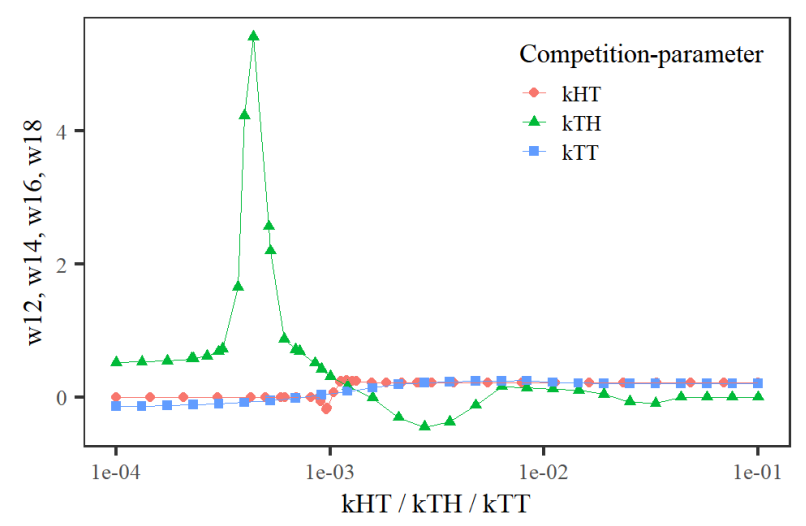

Figure 9: Behavior of the final perceptron weights of the even pattern-vector components in dependency of the three critical competition parameters between host- and tumor-tissue.

Furthermore, the importance of the antigen-pattern vector was evaluated by assigning various antigen patterns to the final tumor subpopulation $T_{32}$. Depending on the number of assigned antigens and subsequent elimination of the corresponding antigen-bearing tumor cells, the tumor cell population with the smallest amount of antigen pattern vector components dominates the competition and reaches a steady state which is dependent on the strength of immune system mediated elimination (complete remission was not observed in this setting).

The investigation of other mutation trees with different branching of sub-populations didn't exhibit fundamental changes in the dynamic behavior.

\section{Discussion and Conclusion}

The presented model is describing an artificial system and may include partially the semantics of a biological system. The matching with a real-world tumor-immune ecosystem (in patient) is questionable and the chosen approach may be too simplistic. However, the idea of an immune system considered as a trainable perceptron offers new hypothesis for novel approaches to anti-cancer treatments, treatments of infectious diseases or even vaccination. In principle, this idea is in agreement with the hygiene hypothesis (Sironi and Clerici, 2010). In this work, we demonstrated the feasibility of combining a tumor ecosystem with a learning immune system. In principle, the observed dynamics can be understood by looking to the development of the cell populations, the generation of the danger signal and the way how the perceptron is trained. An interesting feature is the evolution into negative weights for the host tissue. This separation to the tumor cell population is not only visible for the orthogonal setting for the antigen pattern vectors. The negative weights reduce the perceptron response $Y$ and could therefore be considered as an immune-suppressive factor. But the tumorassociated odd weights compensate this effect partially by their increasing values (figure $4 \mathrm{~b}$ ) which lead to an immune response as soon as the tumor cell populations increase. In this light, it would be interesting to expand the model to a multi- 
compartmental model, where some compartments contain only host tissue or small tumor cell populations (metastases). However, reinforced learning of the artificial adaptive immune system seems to lead to a response which is strongly dependent on the ecosystem dynamics. If this would apply to a real patient, RT fractionation schemes should be adapted to the dynamic response of the tumor-host-tissue system.

Some aspects of the real immune system are not yet implemented. One component which may have an important influence to the evolution of the ecosystem is the presence of memory cells. In the real biological system, memory cells lead to a faster and more pronounced activation of the immune response after a first appearance of the antigens and may modify speed and effectivity of the reinforced learning process. The implementation of such a memory function may lead to a more effective suppression of the tumor after RT application. Regarding repopulation of tumor cells during therapy as a cause of treatment failure (Kim and Tannock, 2005), the investigation of different scenarios avoiding the evolution of non-detectable tumor cells $\left(T_{32}\right)$ by a more effective suppression of progenitor cells would be interesting.

The system is described by a model using ordinary differential equations. This allows a straightforward implementtation of a perceptron with reinforced learning leading to a simplistic description of the immune system. As an alternate approach, agent-based modelling may be used for tumor (Zhang et al., 2009) and immune cell - mediated response.

The applicability of the presented system to clinical treatment optimization is not possible and may remain strongly limited when refined (e.g. memory, more appropriate parameter estimates). A step forward would be possible as soon as methods for time-resolved monitoring of the immune status of patients are available. Repeated acquisition of biopsy material, blood samples and other invasive sampling are not feasible in clinical treatments of human patients but may be considered for animal patients bearing spontaneous tumors. However, imaging modalities such as PET-scans or MRI may help to get time-resolved information about tumor size, metabolism etc. and may be used for accessing at least some aspects of the tumor ecosystem dynamics.

\section{References}

Baumann, M., Krause, M., Overgaard, J., Debus, J., Bentzen S.M., Daartz J. et al. (2016), Radiation oncology in the era of precision medicine. Nat Rev Cancer, 16(4):234-249.

Banda P, Teuscher C, Lakin M.R. (2013): Online learning in a chemical perceptron. Artificial Life 19, 195-219.

Basanta, D., Anderson, A.R.A. (2015). Exploiting ecological principles to better understand cancer progression and treatment. Interface Focus, 3:20130020. http://dx.doi.org/10.1098/rsfs.2013.0020

Basanta, D., Gatenby, R.A., and Anderson, A.R. (2012). An exploiting evolution to treat drug resistance: combination therapy and the double bind. Mol. Pharmaceut., 9:914-921.

Chen, K.W., and Pienta, K.J. (2011). Modeling invasion of metastasizing cancer cells to bone marrow utilizing ecological principles. Theoretical Biology and Medical Modelling, 8:36.

Crespi, B., and Summers, K. (2005). Evolutionary biology of cancer. Trends Ecol Evol, 20:545-552. (doi:10.1016/j.tree.2005.07.007)

Daugaard, M., Rohde, and M., Jaattela, M. (2007). The heat shock protein 70 family: Highly homologous proteins with overlapping and distinct functions. FEBS letters. 2007, Jul 31;581(19):3702-10.
Dunn, G.P., Bruce, A.T. Ikeda, H. Old, L.J. Schreiber, R.D. (2002). Cancer immunoediting: from immunosurveillance to tumor escape. Nat. Immunol., 3, 991-998, doi:10.1038/ni1102-991.

Fridman, W.H. Zitvogel, L. Sautès-Fridman, C. Kroemer, G. The immune contexture in cancer prognosis and treatment. Nat. Rev. Clin. Oncol. 2017, 14, 717-734, doi:10.1038/nrclinonc.2017.101.

Gatenby, R.A., Gillies, R.J., and Brown, J.S. (2010). The evolutionary dynamics of cancer prevention. Nat. Rev. Cancer, 10:526-527. (doi:10.1038/nrc2892)

González-García, I., Solé, R. V., and Costa, J. (2002). Metapopulation dynamics and spatial heterogeneity in cancer. Proc. Natl. Acad. Sci., U.S.A. 99:13085-13089.

Grimsley, C., and Ravichandran K.S. (2003). Cues for apoptotic cell engulfment: Eat-me, don't-eat-me, and come-get-me signals. Trends in Cellular Biology, 9:465.

Horsman, M.R., Mortensen, L.S., Petersen, J.B., Busk, M., and Overgaard J. (2012). Imaging hypoxia to improve radiotherapy outcome. Nat. Rev. Clin. Oncol., 9(12):674-687.

Kim, J.J., and Tannock, I.F. (2005). Repopulation of cancer cells during therapy: an important cause of treatment failure. Nature Reviews Cancer, 5:517-525.

Matzinger P (2002): The danger model: a renewed sense of self. Science 296,(5566): 301-305

Mehrara, E., Forssell-Aronsson, E., Ahlman, H., and Bernhardt, P. (2007). Specific growth rate versus doubling time for quantitative characterization of tumor growth rate. Cancer Res., 67(8):39703975.

Merlo, L.M.F., Pepper, J.W., Reid, B.J., and Maley, C.C. (2006). Cancer as an evolutionary and ecological process. Nat. Rev. Cancer, 6:924935. (doi:10.1038/nrc2013)

Pienta, K.J., McGregor, N., Axelrod, R., and Axelrod, D.E. (2008). Ecological Therapy for Cancer: Defining Tumors Using an Ecosystem Paradigm Suggests New Opportunities for Novel Cancer Treatments. Translational Oncology, 1:158-164.

Scheidegger S, Fellermann H (2019): Optimizing Radiation Therapy Treatments by Exploring Tumour Ecosystem Dynamics in - silico. In: Fellermann H., Bacardit J., Goñi-Moreno A. \& Füchslin R. M., editors, ALIFE 2019: The 2019 Conference on Artificial Life, pages 236-242. The MIT Press Journals, https://doi.org/ 10.1162 /isal a 00167

Scheidegger S, Fuchs HU, Füchslin RM (2014): Computational Methods for Exploring the Dynamics of Cancer: The Potential of State Variables for Description of Complex Biological Systems. Proc. NOLTA 2014, 168-171.

Scheidegger, S, Lutters, G, and Bodis, S (2011a). A LQ-based kinetic model formulation for exploring dynamics of treatment response of tumours in patients. Z. Med. Phys., 21:164-173.

Scheidegger, S., and Füchslin, R.M. (2011b). Kinetic model for dose equivalent - an efficient way to predict systems response of irradiated cells. Proc. of ASIM 2011, (full papers, ISBN 978-3905745-44-3)

Sironi M., Clerici M. (2010): The hygiene hypothesis: An evolutionary perspective. Microbes and Infections 12, 421

Steel, G. G. (1977). The Growth Kinetics of Tumours. Clarendon, Oxford.

Srivastava, S. (2002). Roles of heat-shock proteins in innate and adaptive immunity, Nature Reviews Immunology, 2:185-194.

Thorwarth, D. (2017). Biologically adapted radiation therapy. Z Med Phys, 28:177-183.

Van Leeuwen, C.M., Oei, A.L., Crezee, J., Bel, A., Franken, N.A.P, Stalpers, L.J.A., and Kok, H.P. (2018). The alpha and beta of tumours: a review of parameters of the linear-quadratic model derived from clinical radiotherapy studies. Radiation Oncology, 13:96, doi:10.1186/ s13014-018-1040-z

Vesely, M.D., Kershaw, M.H., Schreiber, R.D., and Smyth, M.J. (2011). Natural innate and adaptive immunity to cancer. Annual Review of Immunology, 29:235-271.

Zhang, L., Wang, Z., Sagotsky, J. A., and Deisboeck, T. S. (2009). Multiscale agent-based cancer modeling. J. Math. Biol., 58:545559 . 